\title{
Influence of Variable Time Operations in Static Instruction Scheduling ${ }^{\star}$
}

\author{
Patricia Borensztejn ${ }^{1}$, Cristina Barrado ${ }^{2}$, and Jesus Labarta ${ }^{2}$ \\ 1 Dep.de Computación, UBA, Argentina. patricia@dc.uba.ar \\ 2 Dep. de Arquitectura de Computadores, UPC, España
}

\begin{abstract}
Instruction Scheduling is the task of deciding what instruction will be executed at which unit of time. The objective is to extract maximum instruction level parallelism for the code. Compilers designed for VLIW and EPIC architectures do static instruction scheduling in a back-end pass. This pass, known as scheduler, needs to have full knowledge of the execution time of each instruction. But memory access instructions have a variable latency, depending on their locality and the memory hierarchy architecture. The scheduler must assume a constant value, usually the execution time assigned to a hit. At execution a miss may reduce the parallelism because idle cycles may appear before the instructions that need the data. This paper describes a statistic model to evaluate how sensitive are the scheduling algorithms to the variable time operations. We present experimental measures taken over two static scheduling algorithms based on software pipelining.
\end{abstract}

\section{Introduction}

Compilers for VLIW, EPIC and superscalar architectures include instruction scheduling as an optimization pass with the objective of minimizing the number of cycles, this is, maximizing parallelism. A special approach that schedules instructions at the loop level is software pipelining [3, 5], where instructions belonging to different iterations of the loop overlap. The Initiation Interval, II, is the number of cycles between the initiation of successive iterations. This value is limited by the data dependencies between loop instructions and by the resource constraints of the actual machine. The main goal of different implementations of software pipelining is to achieve a loop kernel with the minimum $I I$.

The scheduler must have full knowledge of the latencies to schedule instructions. But load and store operations take a variable number of cycles to execute depending on what level of the memory hierarchy holds the values, the buses bandwidths, etc. Schedulers assume a constant value, usually the execution time of a hit. At execution a miss may reduce the parallelism because idle cycles may appear before the instructions that need the data.

This paper presents a statistic model to evaluate the sensitivity of static schedulings to variable time operations (here the memory accesses). Section 2

\footnotetext{
* This work has been supported by the Ministry of Education of Spain under contracts TIC98-0511 and by the MHAOTEU Esprit Project
}

P. Amestoy et al. (Eds.): Euro-Par'99, LNCS 1685, pp. 213216 1999

(C) Springer-Verlag Berlin Heidelberg 1999 
describes the statistic model proposed to evaluate the $I I$, section 3 presents the statistic measures of two software pipelining methods and the last section summarizes the results and the future work.

\section{The Variable Time Model}

This section introduces the Probable $I I$ value, which gives the mean value of the II on the parallel execution. 3] presents how to compute its lower bound:

$$
M I I=\operatorname{Max}(\operatorname{Rec} M I I, \operatorname{Res} M I I)
$$

this is, the $I I$ must preserve data dependences that form recurrences (RecMII) and can use only the available resource (ResMII). RecMII can be computed as maximum ratio distance/weight of the edges for all recurrences and ResMII as the maximum ratio used/available of cycles for all resources.

The increase of the latency of an operation (i.e. miss on a load) on the critical path influences more on parallelism than the increase of an operation that is not on the critical path. Our model takes into account all the combinations of misses and hits of the memory access instructions of the loop body.

Given the dependence graph of a loop, we define a statistical model where the following conditions are hold: (1) Each memory instruction hits with a probability of $P$ and misses with a probability of $1-P,(2)$ the execution time of a memory instruction is $h$ cycles if it hits and $m$ cycles if it misses, with $h<m$, and (3) each instruction hits or misses with independence of the rest of the memory instructions.

Assuming that $L$ is the number of load/store instructions of a loop , $S_{i}$, then there exists $2^{L}$ combinations of hits and misses. We define each combination as an event. Each event has a probability of occur, $P_{r o b} b_{\text {event }}$, that can be calculated as in 2. We define the Probable Initiation Interval, $I I_{\text {Prob }}$, as the mean value of the of the $I I$ of each event weighted by the probability of each event.

$$
\text { Prob }_{\text {event }}=\prod_{i=1}^{L} p_{i} \quad \text { where } p_{i}=\left\{\begin{array}{llll}
P & \text { if } S_{i} & \text { hits } \\
& & \\
1-P & \text { if } S_{i} & \text { misses }
\end{array}\right.
$$

\section{Measures of the Influence of Variable Time Operations}

In this section we present the experimental measures of the influence of variable time operations (the memory accesses) on the expected speedup of software pipelined loops using two different implementations. The variable time operations are all memory accesses: Latency is 1 cycle on cache hit and 10 cycles on cache miss.

The two software pipelining heuristics applied were Hypernode Reduction Modulo Scheduling[4, HRMS, and Graph Traverse Software Pipelining[1], GTSP. HRMS is a modulo scheduling based heuristic. Its objective is 

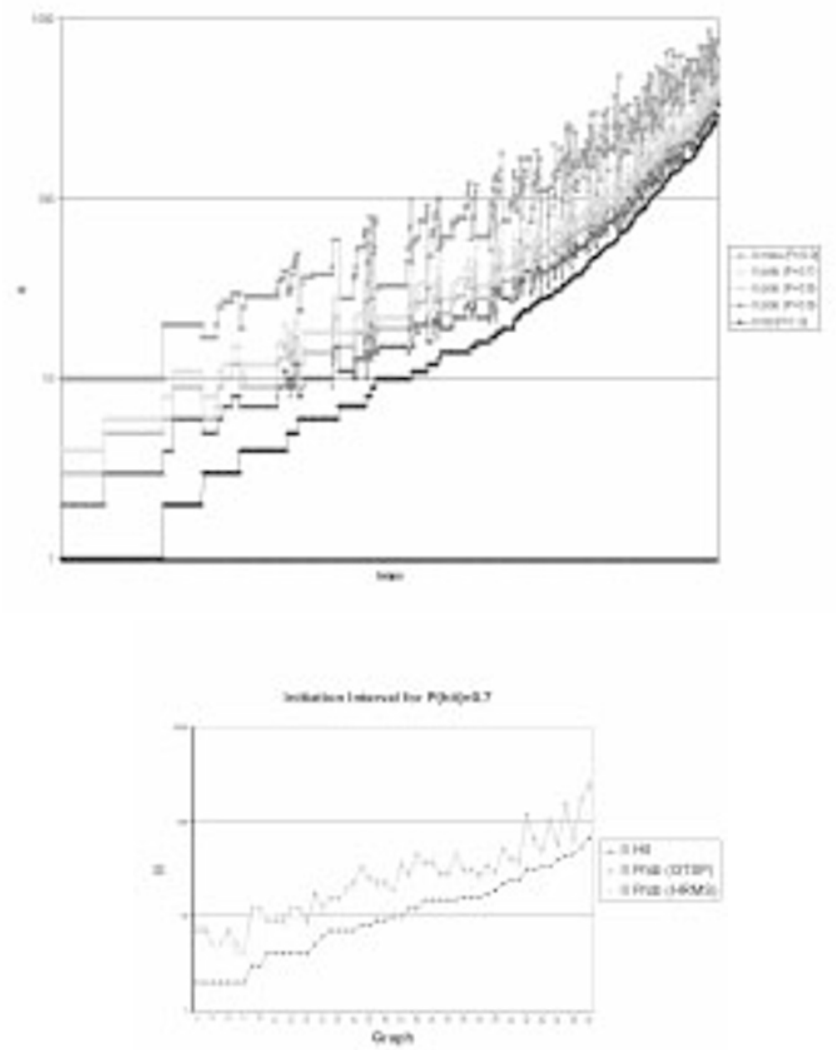

Fig. 1. a) Effects of Variable Latency on II, b) Comparison of two Schedulers

to obtain the minimum $I I$ using the minimum number of registers. GTSP is a graph transformation heuristic targeted to minimize the $I I$.

The methodology used first computes the dependence graphs, DG, of up to 1258 innermost loops of the Perfect Benchmark Club. Each DG is then software pipelined and represented by a Scheduling Graph, SG. The SG [2] is an extension of the DG where new edges are added to introduce the scheduling restrictions. The variable time model is then applied to each SG and the IIprob evaluated for the given hit probability.

Figure 1 1 a shows the IIprob of all the loops assuming five hit probabilities and scheduling with HRMS. The plots are sorted in increasing order of the $I I$. The plot labeled II hit shows the best $I I$ (with a $100 \%$ of memory hits). In the other side the plot $I I$ miss shows the worst $I I$, this is, when every memory access is a miss while the scheduler considered only the hit latency. In between the two bounds the figure shows three more plots, which correspond to intermediate and more realistic situations (hit probabilities of 70\%, $80 \%$ and 90\%). The figure 
shows that the difference between the plots $P=1.0$ and $P=0.9$ is larger than the differences between the plots $P=0.9$ and $P=0.8$. This shows the importance of the first small variations of the hit probability and that speedup penalties comes out even for the small miss ratios.

Figure 1 b compares the sensitivity of the two heuristics for some loops that gave the same II hit. For a hit probability of $70 \%$ the it II prob is, in general, greater for GTSP than for HRMS. Numbers show that one scheduler is more sensitive to variable time operations than the other for loops that a priori they were equal. We investigated the cause of such difference and found that GTSP used only one memory unit when possible, while HRMS used most of the time the two memory units.

\section{Conclusions}

In this paper we show the important influence of variable time operations in the runtime performance of static scheduled loops. We present a statistic model to measure the probable mean parallelism of a loop for a given hit probability. The model shows that the use of the hit latency for memory operations may degrade the application speedup at runtime with a factor equal to the miss latency. Another important outcome is that the performance degradation is not linear. The increment of the $I I_{\text {Prob }}$ is higher when the hit probability goes from $100 \%$ to $90 \%$ than when it decrements from $90 \%$ to $80 \%$.

We show that static schedules are not equally sensitive to variable time operations. One of the objectives of scheduling heuristics should be to minimize of variable time operations sensitivity. We believe that this could be more important for performance than other considerations like minimizing register pressure.

A direct application of our model could be to introduce it into the compiler in order to help choosing the best scheduling considering their $I I_{\text {Prob }}$ Apply statistic regression in a future work will help for fast computing the Probable Initiation Interval.

Also the model can be extended to consider different hit probabilities for the loop memory operations. This, together with a static locality analysis, can be very useful to achieve realistic $I I$ at compile time.

\section{References}

[1] C. Barrado and J. Labarta: "Hamiltonian Recurrence for ILP", EuroPar, 1997.

[2] P. Borensztejn, C. Barrado and J. Labarta: "The Scheduling Graph in the Variable Time Model". Report UPC-DAC-1999-18.

[3] M. Lam: "Software Pipelining: An Effective Scheduling Technique for VLIW machines", Proceedings SIGPLAN'88 PLDI, pp.318-28, 1988.

[4] J. Llosa, M. Valero, E. Ayguadé and A. González: "Hypernode Reduction Modulo Scheduling", Micro-28, pp.350-360, Dec. 1995.

[5] B. Rau: "Iterative Modulo Scheduling: An Algorithm for Software Pipelining Loops", IEEE Micro-27, pp.63-74, Nov. 1994. 